ORIGINAL ARTICLE

\title{
Cryotherapy for acute ankle sprains: a randomised controlled study of two different icing protocols
}

C M Bleakley, S M McDonough, D C MacAuley

See end of article for authors' affiliations

.....................

Correspondence to: Professor McDonough, Health and Rehabilitation Sciences Research Institute, University of Ulster, Jordanstown BT37 OQB, County Antrim, Northern Ireland, UK; $s$. mcdonough@ulster.ac.uk

Accepted 28 March 2006 Published Online First 12 April 2006

\begin{abstract}
Background: The use of cryotherapy in the management of acute soft tissue injury is largely based on anecdotal evidence. Preliminary evidence suggests that intermittent cryotherapy applications are most effective at reducing tissue temperature to optimal therapeutic levels. However, its efficacy in treating injured human subjects is not yet known.

Objective : To compare the efficacy of an intermittent cryotherapy treatment protocol with a standard cryotherapy treatment protocol in the management of acute ankle sprains.

Subjects: Sportsmen $(n=44)$ and members of the general public $(n=45)$ with $\mathrm{mild} / \mathrm{moderate}$ acute ankle sprains.

Methods: Subjects were randomly allocated, under strictly controlled double blind conditions, to one of two treatment groups: standard ice application $(n=46)$ or intermittent ice application $(n=43)$. The mode of cryotherapy was standardised across groups and consisted of melting iced water $\left(0^{\circ} \mathrm{C}\right.$ ) in a standardised pack. Function, pain, and swelling were recorded at baseline and one, two, three, four, and six weeks after injury. Results: Subjects treated with the intermittent protocol had significantly $(p<0.05)$ less ankle pain on activity than those using a standard 20 minute protocol; however, one week after ankle injury, there were no significant differences between groups in terms of function, swelling, or pain at rest.

Conclusion: Intermittent applications may enhance the therapeutic effect of ice in pain relief after acute soft tissue injury.
\end{abstract}

A nkle injuries are one of the most common acute soft tissue injuries, ${ }^{1}$ incurring a significant cost to both the individual and society. ${ }^{2}$ Despite the high incidence, cost, and long term morbidity, however, the optimal method of managing acute ankle sprains remains controversial. ${ }^{2}{ }^{3}$

The application of ice after ankle sprain is accepted clinical practice even if the strength of evidence supporting the use of cryotherapy in management of acute soft tissue injury is generally poor. ${ }^{45}$ Most randomised, controlled trials have focused on post-surgical patients, and have serious flaws in their study design and implementation. In addition, no study has rigorously compared the effectiveness of two different icing protocols; therefore there is no evidence to suggest an optimal mode, duration, or frequency of ice application.

In the absence of robust evidence from clinical studies, one systematic review ${ }^{6}$ made recommendations for an optimal treatment protocol, using evidence derived from investigations on animal models or healthy human volunteers. The evidence suggested that intermittent 10 minute applications were most effective at reducing both skin and deep tissue temperature to optimal therapeutic levels, and melting iced water was deemed the safest and most efficient method of application. ${ }^{6}$ Although this provides preliminary evidence based recommendations for an optimal protocol, its efficacy in treating injured human subjects is not yet known.

The aim of this investigation was to study the effectiveness of cryotherapy in treating acute soft tissue injuries. The specific objective was to compare the efficacy of an intermittent cryotherapy treatment protocol with a standard cryotherapy treatment protocol in the management of acute ankle sprains.

\section{METHODS}

Participants and recruitment

Recruitment began in the University of Ulster in January 2002 and was extended to the Royal Victoria Hospital, Belfast in March 2002. To be eligible for inclusion in the study, subjects had to have sustained a mild/moderate ankle sprain within the preceding 48 hours. A three point grading scale ${ }^{7}$ was used to determine the severity of the sprain. Subjects testing positive on the anterior drawer test or talar tilt test were automatically excluded from the trial.

Delayed physical examination for the presence or absence of a ligament lesion gives diagnostic quality equal to that of arthroscopy. ${ }^{8}$ Other exclusion criteria were subjects $<16$ years old, a bony injury, multiple injuries, or the presence of any cold related conditions. ${ }^{9}{ }^{10}$ The exclusion of a bony injury was made using radiography and/or the Ottawa ankle rules classification. ${ }^{11}$ Before entry into the study, all subjects gave informed, written consent and received a written explanation of the trial, which had been approved by the University of Ulster Research Ethics Committee.

\section{Randomisation}

Before the start of the trial, an independent researcher, who had no contact with the study participants or input into the day to day proceedings of the trial, generated the randomisation sequence using computer software. Stratified randomisation was used with two strata: sports and general population. Separate block randomisation sequences were produced for each subset of participants. An allocation ratio of $1: 1$ was used with a block size of $4 .^{12}$

The randomisation schedules for each stratum were transferred into written instructions (standard or intermittent treatment) and placed in sequentially numbered opaque sealed envelopes. These schedules were used to assign sequentially enrolled participants to either intermittent treatment or standard treatment. Neither the primary researcher nor any of the those involved in treatment allocation were involved in any aspect of randomisation.

The primary investigator, who remained blinded to group assignment until completion of data collection and analyses, carried out subject recruitment, gained informed consent, 
and assessed all baseline and follow up measurements. Three secondary researchers assigned subjects to either the standard $(\mathrm{n}=46)$ or intermittent $(\mathrm{n}=43)$ group and provided advice on the appropriate cryotherapy treatment and standardised treatment. Group assignment was concealed from the secondary researchers, using opaque sealed envelopes, until subject recruitment and informed consent was completed.

\section{Experimental groups}

The mode of cryotherapy was standardised across groups and consisted of melting iced water $\left(0^{\circ} \mathrm{C}\right)$ in a standard sized pack. ${ }^{13}{ }^{14}$ Plastic ice bags $(20 \mathrm{~cm} \times 20 \mathrm{~cm})$ were completely filled with water, placed in a freezer, and removed when frozen. Before application, the packs were held under hot water for 30 seconds and wrapped in a single layer of standardised towelling (moistened until just dripping wet).

Standard ice application consisted of 20 minutes of continuous ice treatment performed every two hours. This duration of treatment has been recommended in the literature $^{10} 15$ and is also commonly used in the clinical setting. ${ }^{15}$ The intermittent ice group applied ice for $10 \mathrm{~min}$ utes. The pack was then removed, and the ankle was rested at room temperature for 10 minutes. The ice was then reapplied for a further 10 minutes. Again intermittent treatments were repeated every two hours, and both groups continued their respective treatments over the first 72 hours of injury. Previous studies ${ }^{13}{ }^{14}$ have shown that the mode and duration of cryotherapy applied in the intermittent protocol reduces skin temperature to $5^{\circ} \mathrm{C}$ immediately after treatment.

Subjects were responsible for ice pack preparation, and all treatments were self administered. At the time of the trial, subjects were given a verbal explanation of the correct procedure for ice pack preparation and application, which was supplemented with step by step written instructions. Patient compliance was monitored using a treatment diary, which was returned to the secondary researcher one week after the injury. Ankle mobility (ankle circles, 30 repetitions $\times 3$ ), calf stretching (non-weight bearing, 30 second holds $\times$ $5)$, and basic proprioceptive exercises were standardised between groups by means of an advice sheet. Subjects were encouraged to perform these exercises once a day for the first week.

\section{Outcome measurement and follow up procedure}

Function, pain, and swelling were recorded at baseline and one, two, three, four, and six weeks after injury.

(1) The primary outcome measure was subjective ankle function, assessed using Binkley's lower extremity functional scale. ${ }^{16}$ This scale has excellent test retest reliability and good construct validity. ${ }^{16}$

(2) Pain was assessed using a $10 \mathrm{~cm}$ visual analogue scale, marked "no pain" at one end and "worst pain ever" at the other. This form of assessment was considered most appropriate because of its high level of repeatability when used serially on the same patient. ${ }^{17}$

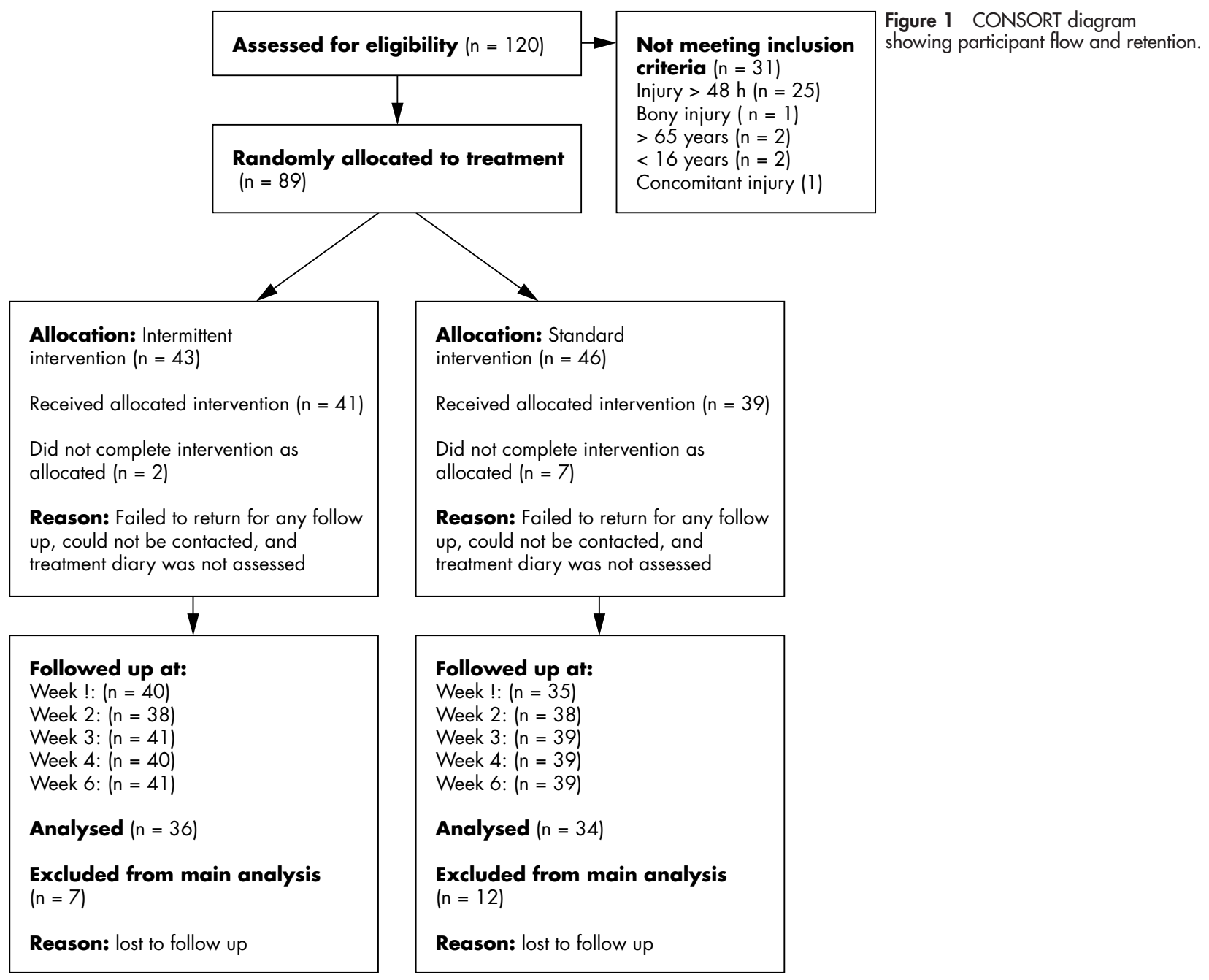




\begin{tabular}{lll|}
\hline $\begin{array}{l}\text { Table } 1 \text { Baseline general and clinical characteristics of patients } \\
\text { two treatment groups }\end{array}$ & \\
\hline & $\begin{array}{l}\text { Intermittent ice } \\
(\mathbf{n}=\mathbf{4 3 )}\end{array}$ & $\begin{array}{l}\text { Standard ice } \\
(\mathbf{n}=46)\end{array}$ \\
\hline Age (years) & $29.8(11.5)$ & $31.2(10.9)$ \\
Height (cm) & $178.8(11.9)$ & $174.3(12.2)$ \\
Weight (kg) & $78.2(12.3)$ & $80.1(9.7)$ \\
Grade 1:grade 2 & $14: 29$ & $11: 35$ \\
Cause of injury (\%) & & 48 \\
Sport & 52 & 13 \\
Work related & 17 & 39 \\
Other & 31 & $27(7.2)$ \\
Time from injury (h)* & $24(6.8)$ & 17 \\
Previous ankle injury (\%) & 15 & 89 \\
Number of patients employed (\%) & 92 & 83 \\
Number of non-smokers (\%) & 79 & $30: 16$ \\
Male:female & $28: 15$ & $22: 24$ \\
Sport:general & $22: 21$ & \\
\hline *Values are mean (SD). & & \\
\hline
\end{tabular}

(3) Swelling was measured using a figure of eight method. All measurements were performed using one quarter inch wide plastic tape following a standard written protocol. ${ }^{18}$ A mean of two measures, from both the injured and uninjured ankle, was recorded. To establish the degree of swelling on the injured side, a difference value was calculated by subtracting the mean value for swelling for the uninjured ankle from the injured ankle. The figure of eight method has been shown to be a highly reliable ${ }^{1920}$ and valid $^{20}$ tool for measuring the girth of both healthy and oedematous ankles.

\section{Power calculations and statistical analysis}

A randomised controlled pilot study $(\mathrm{n}=15)$ was used to determine sample size and recruitment targets. The power analysis calculations based on the results showed that assuming $80 \%$ power and a $0.05 \alpha$ value, and based on a between-within repeated measures analysis of variance design, a minimum number of 40 subjects would be required for each intervention group, to detect a mean difference of 9 (in either direction) on the questionnaire for the primary outcome. ${ }^{16}$ Using information from a previous study ${ }^{21}$ in the same hospital department, a $10 \%$ attrition rate was estimated at each phase of the trial, therefore this study aimed to recruit a total of 128 subjects. Recruitment was planned for a 52 week period, and an average of 2.5 new subjects would be recruited a week.

Data were analysed using the Statistical Packages for the Social Sciences (Windows 9.0).

Descriptive statistics were performed to produce mean (SEM).

For all outcome measures, a within subjects repeated measures of analysis of variance was performed to determine the significant change over time between groups. The raw scores recorded at baseline and week 1, 2, 3, 4, and 6 were the six levels on the within subjects factor. The two treatment groups (intermittent and standard) were the two levels on the between subject factor.

In addition, a within subjects repeated measures analysis of covariance was undertaken to control for the subjects baseline levels before investigation of their change in subsequent time points. Time (five levels: week 1, 2, 3, 4, and 6) was the within subject factor, treatment group (two levels: intermittent and standard) was the between subjects factor, and baseline values were used as the covariate. For both the analysis of variance and analysis of covariance, in cases when Mauchley's test revealed that the assumption of sphericity had been violated $(\mathrm{p}<0.05)$, the Greenhouse-Geisser

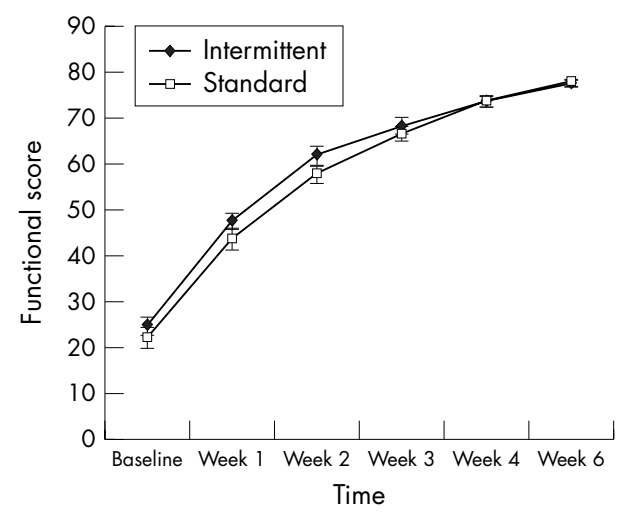

Figure 2 Line graph showing mean (SEM) values for ankle function, assessed using Binkley's lower extremity functional scale, ${ }^{16}$ for intermittent and standard groups at baseline and week 1, 2, 3, 4, and 6 after injury. Values for the interaction time $\times$ group (within subject factor $=$ time; between subject factor $=$ treatment group): $F=1.951 ; \mathrm{df}=5$; df error $=340 ; p=0.85$. Sphericity was assumed, as GreenhouseGeisser's procedure produced a non-significant $F$ ratio.

Table 2 Baseline values of all outcome measures by treatment group

\begin{tabular}{lllll}
\hline & $\begin{array}{l}\text { Intermittent } \\
(\mathbf{n}=\mathbf{4 3})\end{array}$ & $\begin{array}{l}\text { Standard } \\
(\mathbf{n = 4 6 )}\end{array}$ & t Value & p Value \\
\hline $\begin{array}{l}\text { Subjective function (lower extremity } \\
\text { functional scale) }\end{array}$ & $24.6(1.96)$ & $22.3(2.23)$ & 0.9 & 0.38 \\
Pain intensity at rest (10 cm VAS) & $1.0(0.16)$ & $1.7(0.22)$ & 1.7 & 0.08 \\
Pain intensity on activity (10 cm VAS) & $3.9(0.28)$ & $4.7(0.27)$ & 1.1 & 0.3 \\
Swelling (cm) (figure of eight method) & $2.1(0.12)$ & $2.3(0.16)$ & 0.3 & 0.7 \\
\hline
\end{tabular}

Values are mean (SEM). Independent samples $t$ test was used to determine statistical significance $(p<0.05)$. VAS, Visual analogue scale. 
procedure was applied to adjust for the degrees of freedom. The level of significance for all tests was set at 0.05.

In the event of any significant interaction between group and time, a univariate analysis of covariance was undertaken. This highlighted the time point where the significant differences lay. For multiple comparisons, Bonferroni adjustments were applied and the p values were lowered accordingly. Initially, all analyses were undertaken using listwise deletion and then repeated using the expectation maximisation algorithm method to impute missing values..$^{22}$

\section{RESULTS}

Eighty nine subjects (male:female, 58:31; mean (SD) age 29.9 (10.32) years) with acute ankle sprains were recruited from the staff and student population at the University of Ulster and the Royal Victoria Hospital, United Hospitals Trust, between January 2002 and February 2003. Although the original target was to randomise 128 subjects, the attrition rate was lower than expected, and recruitment was stopped prematurely. Participant flow and retention are summarised in the CONSORT diagram (fig 1). Table 1 shows the basic and clinical characteristics of patients in each group. Table 2 highlights that at baseline there were no significant differences between groups in terms of ankle function, pain at rest or on activity, or swelling. The degree of compliance in both groups was good: mean (SEM) number of ice treatments over the first 72 hours was $5.7(0.41)$ in the standard group and $5.5(0.42)$ in the intermittent group $(t=0.21, \mathrm{p}=0.84)$.

\section{Intention to treat analysis}

Nineteen subjects were lost to follow up, and therefore full datasets from 70 subjects were available for the intention to treat analysis. All 70 of these patients received the relevant intervention as allocated and all of them complied adequately with treatment. Subjects were deemed to be compliant if they applied ice at least once each day over the first 72 hours, as rated by their patient diaries. None of them were considered protocol violators, and it was not deemed necessary to undertake an additional per protocol analysis. ${ }^{24}$

\section{Function/swelling/pain at rest}

Values for subjective ankle function, swelling, and pain at rest improved over the entire follow up period (figs 2-4). A repeated measures analysis of variance revealed that the improvements over time were significant in both groups $(p<0.05)$ for all outcomes. However, there was no significant interaction between time and group. These findings were

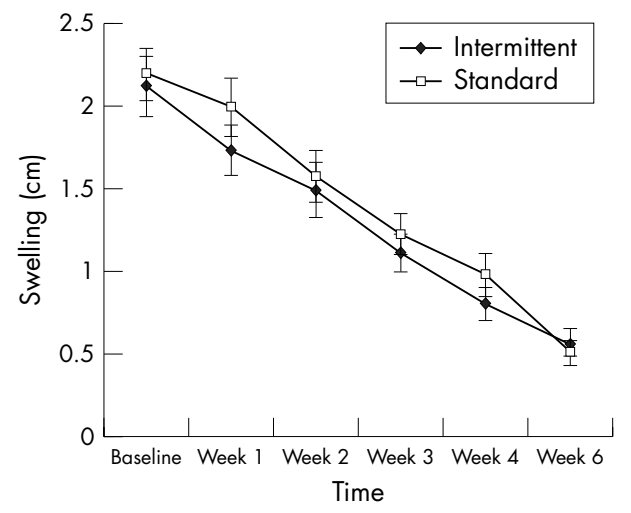

Figure 3 Line graph showing mean (SEM) values for ankle swelling for intermittent and standard groups at baseline and week 1,2,3,4, and 6 after injury. Values for the interaction time $\times$ group (within subject factor $=$ time; between subject factor $=$ treatment group): $F=0.839 ; \mathrm{df}=5$; df error $=340 ; p=0.523$. Sphericity was assumed, as GreenhouseGeisser's procedure produced a non-significant $F$ ratio.

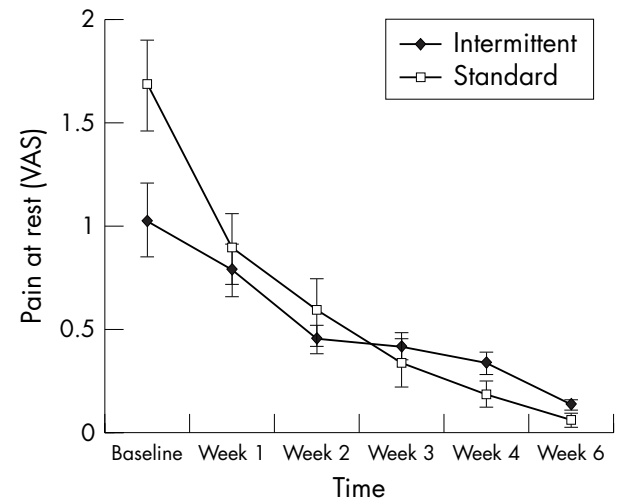

Figure 4 Line graph showing mean (SEM) values for ankle pain at rest for intermittent and standard groups at baseline and week 1, 2, 3, 4, and 6 after injury. Values for the interaction time $\times$ group (within subject factor $=$ time; between subject factor $=$ treatment group): $F=5.486$; $\mathrm{df}$ $=2.456 ; \mathrm{df}$ error $=340 ; \mathrm{p}=0.076$. Degrees of freedom were adjusted using Greenhouse-Geisser's procedure. VAS, Visual analogue scale.

consistent with the repeated measures analysis of covariance, which adjusted for baseline differences.

\section{Pain on activity}

Values in both treatment groups decreased at each time point during the six week follow up (fig 5). A repeated measures analysis of variance revealed that the decreases over time were significant $(p<0.05)$, and there was a significant interaction between group and time $(p<0.05)$.

These findings were consistent with the repeated measures analysis of covariance, which adjusted for baseline differences. A univariate analysis of covariance revealed that the pain values reduced significantly more between baseline and week $1(p<0.017)$ in the intermittent group compared with the standard group. As there were multiple comparisons made, Bonferroni adjustment reduced the $\mathrm{p}$ value to 0.017 for this analysis.

\section{Imputed analysis}

An alternative analysis was conducted to address the problem of dropouts. The expectation maximisation alogarithm method was used to impute missing values. ${ }^{22}{ }^{23}$ This restored

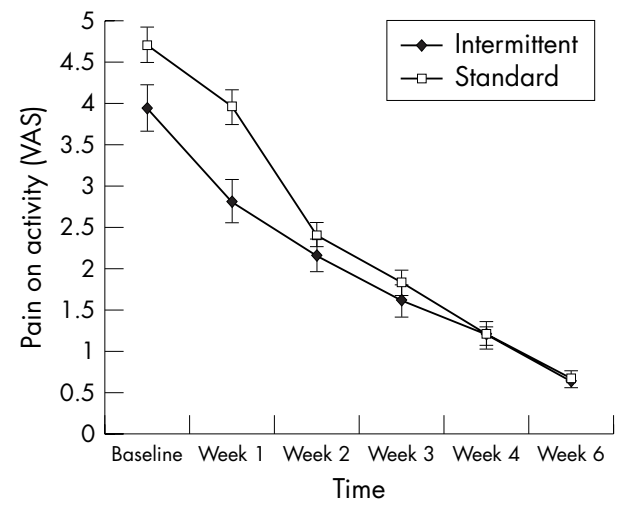

Figure 5 Line graph showing mean (SEM) values for ankle pain on activity for intermittent and standard groups at baseline and week 1, 2, 3,4 , and 6 after injury. Values for the interaction time $\times$ group (within subject factor $=$ time; between subject factor $=$ treatment group): $F=$ 4.292; $\mathrm{df}=3.152 ; \mathrm{df}$ error $=340 ; \mathrm{p}=0.005$. Degrees of freedom were adjusted using Greenhouse-Geisser's procedure. The $p$ value indicates significant interaction. VAS, Visual analogue scale. 
the sample size to its original value level, thus increasing the power of the study $(\mathrm{n}=80)$. The findings from both the repeated measures analysis of variance and adjusted repeated measures analysis of covariance were consistent with the intention to treat analysis.

\section{Adverse effects}

There were no reported incidences of skin burns, nerve palsies, or any other potentially deleterious effects in either group throughout the duration of the trial.

\section{Retrospective power analysis}

In this study, the effect size for the primary outcome measure (subjective function) was calculated at 0.25. Full datasets were obtained from 70 subjects, therefore the power of this study, based on a $0.05 \alpha$ value and a between-within repeated measures analysis of variance design, was calculated at 0.59 . The imputed analysis increased the sample size to $\mathrm{n}=80$, and the power of the study increased to 0.68 .

\section{DISCUSSION}

\section{Overview}

This is the first randomised controlled study to rigorously compare the effectiveness of two different, clinically appropriate cryotherapy protocols, using humans with closed soft tissue injuries. Both groups showed significant improvements over time in pain, function, and swelling. The only significant difference between the two groups was that subjects applying the intermittent protocol had less pain during everyday activity at week 1 .

\section{Cryotherapy and ankle pain}

The mechanisms by which ice decreases pain after injury is a contentious issue: it may reduce nerve conduction ${ }^{25}$ or muscle spasm or have an antinociceptive effect on the gate control mechanism. ${ }^{26}$ There is some evidence that cold induced analgesia is short term, however, continuing for just 1530 minutes after treatment cessation. ${ }^{27}$ In this study, ice application was confined to the acute phases of injury, and it is unlikely that analgesia by any of these proposed mechanisms ${ }^{26}$ could directly influence ankle pain, weeks or even days outside this time period. Potentially, however, the degree of cooling achieved in the acute phases may indirectly influence the levels of ankle pain one week after injury, which may explain the significantly lower levels reported in the intermittent group. For example, as intermittent 10 minute applications maintain tissues at optimal levels of $10-15^{\circ} \mathrm{C}$ for longer than standard 20 minute treatments, ${ }^{6}$ subjects using this protocol would also reduce metabolism to optimal levels for longer. This may have reduced the degree of secondary cell injury, thereby minimising the magnitude of the inflammatory response. ${ }^{28}$ This may also be associated with reduced swelling, nerve damage, muscle spasm, and cell mediators, all of which may influence subjective pain after ankle injury. ${ }^{29}$

In addition, by providing optimal cooling, intermittent ice applications $^{6}$ provide more efficient short term analgesia. Again, although this would not directly influence ankle pain at the first follow up, it may have allowed earlier mobilisation. ${ }^{9}$ There is much evidence that controlled mobilisation aids soft tissue healing ${ }^{30}$ and is associated with enhanced collagen fibre growth and realignment ${ }^{31}$ and less soft tissue fibrosis, and therefore a decreased likelihood of pain. Moreover, one might postulate that the very nature of an intermittent treatment may have been more conducive to performing therapeutic exercise. Anecdotal recommendations state that 10 minutes of cryotherapy can induce sufficient analgesia to allow therapeutic exercise, ${ }^{25}$ and much of the scientific evidence suggests that superficial tissues reach their peak temperature reduction after about 10 minutes of cooling. ${ }^{6}$ Therefore, in effect, the intermittent group may have had two opportunities to exercise and mobilise their ankle in a pain-free state (at the interim period between treatments and after the second 10 minute treatment) compared with just one in the standard group (at the end of the 20 minute treatment).

\section{Ankle function}

This study found no significant difference in functional status between groups. However, a large number of subjects in both groups had difficulty running on uneven ground and making cutting movements when running six weeks after the injury, despite recording low levels of pain on activity. The reason for this persistent difficulty with functional tasks may have been functional instability rather than pain. ${ }^{32}$ Functional instability has been defined as the occurrence of recurrent joint instability or the sensation of joint instability because of contributions of any neuromuscular deficits. ${ }^{32} \mathrm{~A}$ component of its pathogenesis has often been attributed to proprioceptive deficits. ${ }^{33}$ However, further study is required to assess the extent that proprioception (or an element of proprioception) influences subjective ankle function.

\section{Ankle swelling}

In all cases in this study, ankle sprain resulted in an increase in ankle girth. Such post-traumatic oedema may compound the extent of tissue damage, delaying healing, and can even result in some degree of chronic disability. ${ }^{34}$ Cold is thought to cause vasoconstriction, decreasing the permeability of local blood vessels, thereby reducing the degree of haemorrhage ${ }^{35}$ after injury. Dolan et $a l^{36}$ recorded decreases in animal limb girth after cooling, whereas a number of animal ${ }^{37} 38$ and human $^{39}$ studies have reported net increases in tissue girth immediately after cryotherapy. This increase in swelling has been attributed to cold induced damage to superficial lymph vessels, ${ }^{40}$ rather than cold induced vasodilation. In the present study, swelling decreased steadily over the six weeks after injury in both groups, and, although this occurred at slightly different rates, there were no significant differences between groups. Although it seems that neither the intermittent nor standard protocols caused an increase in limb girth, these measurements were recorded at least four days after ice application, and the immediate post-treatment effects could not be assessed.

\section{Study limitations}

The retrospective analysis revealed that the power of the study was 0.68 . This fell below the expected level $(80 \%)$, and there is an increased risk of type 2 error. ${ }^{41}$ The only significant difference between groups was that the degree of pain on activity was significantly lower in the intermittent group at week 1. Generally, however, the overall pain scores on activity were low, and, at week 1 , both the intermittent group and standard group recorded mean values of less than 5/10. Indeed, these values agree with other studies, ${ }^{42}$ which also indicate that the degree of pain after ankle sprain is moderate to low. Others ${ }^{43}$ have postulated that, in certain instances, the differences between groups in visual analogue scale score may have no clinical significance, even if they achieve statistical significance.

\section{CONCLUSION}

The application of an intermittent cryotherapy protocol after mild or moderate ankle sprain significantly reduced the level of subjective pain on activity, one week after the injury, compared with a standard protocol. There was no significant difference in terms of function, swelling, or pain at rest. It appears that intermittent applications may enhance the 


\section{What is already known}

- Cryotherapy is largely accepted as the treatment of choice for acute soft tissue injury, but current recommendations have many shortcomings and selection of parameters in a clinical environment continues to be made pragmatically

- There is preliminary evidence that shorter intermittent applications are most effective at reducing tissue temperature

\section{What this study adds}

- This is the first randomised controlled study to stringently compare two different cryotherapy protocols using subjects with closed soft tissue injury

- In accordance with basic science research, the results highlight that shorter, intermittent applications may enhance the analgesic effect of ice after acute ankle injury

therapeutic effect of ice after acute soft tissue injury, but the benefits are currently restricted to pain relief in the early stages of rehabilitation.

\section{Authors' affiliations}

C M Bleakley, S M McDonough, Health and Rehabilitation Sciences Research Institute, University of Ulster, Jordanstown, County Antrim, Northern Ireland, UK

D C MacAuley, Hillhead Family Practice, Belfast, Northern Ireland, UK Competing interests: none declared

\section{REFERENCES}

1 Garrick JG, Requa RK. The epidemiology of foot and ankle injuries in sports. Clin Podiatr Med Surg 1989;6:629-37.

2 de Bie RA, de Vet HC, van den Wildenberg FA, et al. The prognosis of ankle sprains. Int J Sports Med 1997;18:285-9.

3 Shrier I. Treatment of lateral collateral ligament sprains of the ankle: a critical appraisal of the literature. Clin J Sport Med 1995;5:187-95.

4 Bleakley CM, McDonough SM, MacAuley DC. The use of ice in the treatment of acute soft tissue injuries. A systematic review of randomized controlled trials. Am J Sports Med 2004;32:251-61.

5 Bleakley CM, MacAuley DC, McDonough SM. How good is ice in the treatment of acute soft tissue injuries? In MacAuley D, Best T, eds. Evidence based sports medicine, 2nd ed. Oxford: Blackwell BMJ books, 2006, in press.

6 MacAuley D. Ice therapy: how good is the evidence? Int J Sports Med $2001 ; 22: 379-84$.

7 Rourke K. The evaluation and treatment of acute ankle sprains. J Emerg Med 1994;20:528-35.

8 Van Dijk CN, Lim LSL, Bossuyt PMM, et al. Physical examination is sufficient for the diagnosis of sprained ankles. J Bone Joint Surg [Br] 1996;78:958-62.

9 Knight KL. Cryotherapy in sports injury management. International Perspectives in Physiotherapy 1989:4:163-85.

10 Swenson C, Sward L, Karlsson J. Cryotherapy in sports medicine. Scand J Med Sci Sports 1996;6:193-200.

11 Steill IG, McKnight RD, Greenberg GH, et al. Implementation of the Ottawa ankle rules. JAMA 1994;271:827-32.

12 Altman DG, Bland JM. How to randomise. BMJ 1999;319:703-4.

13 Ebrall P, Moore N, Poole R. An investigation of the suitability of infrared telethermography to determine skin temperature changes in the human ankle during cryotherapy. Chiropractic Sports Medicine 1989;3:111-19.

14 Ebrall PS, Bales GL, Frost BR. An improved clinical protocol for ankle cryotherapy. Journal of Manual Medicine 1992;6:161-5.

15 Kerr KM, Daily L, Booth L. Guidelines for the management of soft tissue (musculoskeletal) injury with protection, rest, ice, compression and elevation (PRICE) during the first 64 hours. London: Chartered Society of Physiotherapy, 1999.

16 Binkley J. The lower extremity functional scale (LEFS) Scale Development, Measurement Properties, and Clinical Application. Phys Ther 1999;79:371-83
17 Katz J, Melzack R. Measurement of pain. Surg Clin North Am 1999;79:231-52.

18 Mawdsley RH, Hoy DK, Erwin PM. Criterion-related validity of the figure of eight method of measuring ankle edema. J Orthop Sports Phys Ther 1992:30:149-53.

19 Peterson EJ, Irish SM, Lyons CL, et al. Reliability of water volumetry and the figure of eight method on subjects with ankle joint swelling. J Orthop Sports Phys Ther, 1999;29, 609-15.

20 Mawdsley RH, Hoy DK, Erwin PM. Criterion-related validity of the figure-ofeight method of measuring ankle edema. J Orthop Sports Phys Ther 2000;30: 149-53

21 Minder PM. Musculoskeletal pain and dysfunction: Effects of phototherapeutic low intensity monochromatic infrared radiation and electro physical agents. DPhil thesis, University of Ulster, 2002.

22 Arbuckle JL. Full information estimation in the presence of incomplete data. In Marcoulides GA, Schaumaker RE, eds. Advanced structural equation modelling: issues and techniques, Mahwah, NJ; Lawrence Erlbaum Associates, 1996:245-77.

23 Duncan TE, Duncan SC, Strycker LA, et al. An introduction to latent variable growth modelling: concepts, issues and applications. London: Lawrence Erlbaum Associates, 1999.

24 Sim J, Wright C. Research in health care: concepts, designs and methods. Cheltenham: Stanley Thornes Publishers Ltd, 2000.

25 Knight KL, Brucker JB, Stoneman PD, et al. Muscle injury management with cryotherapy. Athletic Therapy Today 2000;5:26-30.

26 Ernst E, Fialka V. Ice freezes pain? A review of the clinical effectiveness of analgesic cold therapy. J Pain Symptom Manage 1994;9:56-9.

27 Curkovic B, Vitulic V, Babic-Naglic D, et al. The influence of heat and cold on the pain threshold in rheumatoid arthritis. Z Rheumatol 1993;52:289-91.

28 Merrick MA. Secondary injury after musculoskeletal trauma: a review and update. J Athl Train 2002;37:209-17.

29 Houglum PA. Soft tissue healing and its impact on rehabilitation. Journal of Sports Rehabilitation 1992;1:19-39.

30 Karlsson J, Eriksson BI, Sward L. Early functional treatment for acute ligament injuries of the ankle joint. Scand J Med Sci Sports 1996;6:341-5.

31 Oakes BW. Acute soft tissue injuries. Nature and management. Aust Fam Physician 1981;10:3-16.

32 Hertel J. Functional instability following a lateral ankle sprain. Sports Med 2000;29:361-71

33 Lentell G, Baas B, Lopez D, et al. The contributions of propioceptive deficits, muscle function, and anatomic laxity to functional instability of the ankle. J Orthop Sports Phys Ther 1995;21:206-15.

34 Wilkerson GB. Treatment of the inversion ankle sprain through synchronous application of focal compression and cold. Athletic Training 1991;26:220-237.

35 Karunakara RG, Lephart SM, Pinciverio DM. Changes in forearm blood flow during single and intermittent cold application. J Orthop Sports Phys Ther 1999;29:177-80.

36 Dolan MG, Thorton RM, Fish DR, et al. Effects of cold water immersion on edema formation after blunt injury to the hind limbs of rats. J Athl Train 1997;32:233-7.

37 Farry PJ, Prentice NG, Hunter AC, et al. Ice treatment of injured ligaments: an experimental model. N Z Med J 1980;91:12-14.

38 McMaster WC, Liddle S. Cryotherapy influence on post-traumatic limb edema. Clin Orthop 1980;150:283-7.

39 Cote DJ, Prentice WE, Hooker DN, et al. Comparison of three treatment procedures for minimizing ankle sprain swelling. Phys Ther 1988;68:1064-76.

40 Meeusen R, Lievens P. The use of cryotherapy in sports injuries. Sports Med 1986;3:398-414.

41 Norton BJ, Stube MJ. Understanding statistical power. J Orthop Sports Phys Ther 2001;31:307-15.

42 Campbell J, Dunn T. Evaluation of topical ibuprofen cream in the treatment of acute ankle sprains. J Accid Emerg Med 1994;11:178-82.

43 Farrar JT, Portenoy RK, Berlin JA, et al. Defining the clinically important difference in pain outcome measures. Pain 2000;88:287-94.

\section{COMMENTARY}

This is an interesting paper which addresses a problem that every professional involved in sports medicine will encounter. For how long should I use cryotherapy, and, if I remove the cooling agent for a short period, will the patient experience reactive hyperaemia? Some patients may even report that cooling is a little uncomfortable, and some want to remove the cooling agent for short periods. Will this cause a reaction that may be counter-productive to the rehabilitation of acute ankle injuries? No, and this trial also tells us that cryotherapy is safe. Small periods without ice do not compromise the short term result and may even be more beneficial in the short term.

J Bjordal University of Bergen, Norway; imb@hib.no 